\title{
ETIOLOGÍA DE LA NEUMONÍA ADQUIRIDA EN LA COMUNIDAD \\ Serie de casos del Hospital de San José
}

Sandra L. Suárez. MD*, Henry Tovar. MD**, Andrea C. Piña. MD*** y José Ignacio Hernández. MD****

\section{Resumen}

La neumonía adquirida en la comunidad (NAC) continúa siendo una enfermedad grave. Más de 5.6 millones de casos ocurren al año y cerca de 1.1 millón requieren hospitalización. En el Hospital de San José se encontró durante los dos últimos años un alto porcentaje de NAC como causa de ingreso al servicio de medicina interna (37.5\%).

Dadas las implicaciones de la presencia de esta enfermedad, es importante conocer los gérmenes causantes y la sensibilidad a los distintos antibióticos utilizados. Realizamos un estudio descriptivo de una serie de 159 pacientes que ingresaron con diagnóstico de NAC, de abril 1 de 2003 a noviembre 30 de 2005. De ellos, 63 hombres (49.62\%) y 96 mujeres (60.37\%). Se catalogaron 86 como NAC IIIA (54.1\%), 66 como NAC IIIB (41.5\%), 6 NAC IVA (3.8\%) y un paciente como NAC IVB (0.6\%). Se aisló el germen casual en el $\mathbf{8 . 8 \%}$ de las muestras tomadas, el más frecuente Klebsiella pneumoniae (3.1\%), seguido en menor porcentaje por Streptococcus pneumoniae, Staphylococcus aureus, Staphylococcus hominis y Pseudomona aeruginosa.

Palabras clave: Neumonía, comunidad.

Abreviaturas: NAC, neumonía adquirida en la comunidad; NAC I, II, III A, III B, IV A, IV B, ver Tabla 2; EPOC, enfermedad pulmonar obstructiva crónica, DM, diabetes melitis, IRC, insuficiencia renal crónica; ICC, insuficiencia cardíaca congestiva.

\section{Introducción}

La NAC continúa siendo una enfermedad grave, a pesar de la disponibilidad de nuevos y potentes antibióticos. En Estados Unidos es la sexta causa de muerte y la primera por enfermedad infecciosa. ${ }^{1,2}$

La neumonía es una infección del parénquima pulmonar que puede ser causada por diversas especies bacterianas, micoplasmas, clamidias, ricketsias, virus, hongos y parásitos. No es, por tanto, una enfermedad única, sino un grupo de infecciones

Fecha recibido: 20 de febrero de 2006

Fecha aceptado: 2 de mayo de 2006

* Instructora Asociada, servicio de medicina interna Fundación Universitaria de Ciencias de la Salud, Hospital de San José.

** Instructor Asistente, servicio de medicina interna Fundación Universitaria de Ciencias de la Salud, Hospital de San José.

*** Residente III de medicina interna, Fundación Universitaria de Ciencias de la Salud.

***** Jefe del Departamento Médico, Profesor Titular Fundación Universitaria de Ciencias de la Salud, Hospital de San José, Miembro de Número de la Sociedad de Cirugía de Bogotá. específicas, cada una con su epidemiología, patogenia, presentación clínica y evolución particular. ${ }^{3} \mathrm{El}$ cuadro clínico más frecuente es la clásica descripción de una neumonía y se resume como la presencia de síntomas inespecíficos como escalofríos, fiebre, tos, disnea, expectoración mucopurulenta y dolor torácico tipo pleurítico acompañados o no de signos de consolidación y estertores finos al examen físico. Cursa con leucocitosis y neutrofilia infiltrados alveolares en la radiografía de tórax, con distribución lobar o multilobar y un germen predominante con la coloración de gram del esputo. ${ }^{4}$ La identificación del microorganismo etiológico es de capital importancia ya que constituye la clave del tratamiento antimicrobiano adecuado. No obstante, debido a la gravedad de la infección, muchas veces se precisa iniciar de inmediato un tratamiento empírico, basado en el contexto en que se adquirió la infección, la presentación clínica, los diferentes hallazgos de la radiografía del tórax, las tinciones de esputo o de otros líquidos corporales infectados y el conocimiento de los patrones actuales de sensibilidad a los tratamientos. ${ }^{4} \mathrm{~A}$ menu- 
do antes de recibir la información del agente causal por el laboratorio y según las recomendaciones de las diferentes guías sobre NAC, todos los pacientes hospitalizados deben recibir terapia antibiótica intravenosa dentro de las primeras ocho horas de su llegada al hospital. ${ }^{3,5}$ En cerca de un tercio de los pacientes es difícil establecer el agente etiológico específico, como sucede cuando no se dispone del esputo para el examen, los hemocultivos son estériles o no existe líquido pleural.

La incidencia de diversos patógenos pulmonares varía según el entorno en que se adquirió la infección (comunidad, residencia de ancianos u hospital). En los pacientes hospitalizados con NAC los más frecuentes son Streptococcus pneumoniae, Haemophilus influenzae, Chlamydia pneumoniae, Legionella pneumophila y Mycoplasma pneumoniae. Por el contrario, se calcula que los bacilos entéricos gram negativos y Pseudomona aeruginosa son causas poco frecuentes de NAC. ${ }^{3,6,7}$

Los pacientes con NAC y con criterios para ser hospitalizados deben someterse a una evaluación microbiológica precoz y a un tratamiento empírico basado en la tinción de gram del esputo y el conocimiento de las sensibilidades a los antimicrobianos de los patógenos pulmonares de la zona geográfica local. ${ }^{3,6,8}$ En el paciente hospitalizado suele ser obligatorio el tratamiento antimicrobiano parenteral. La penicilina o la ampicilina continúan siendo los fármacos de elección para la infección por neumococos sensibles a la penicilina. Según los estudios, la bencilpenicilina en dosis altas por vía intravenosa, la ampicilina, la ceftriaxona ó la cefotaxima representan un tratamiento adecuado en la neumonía por cepas con resistencia intermedia a la penicilina. No obstante, algunas variedades muy resistentes a las cefalosporinas se han convertido en un problema en determinadas zonas geográficas. ${ }^{3}$

En 1960 fueron identificadas las primeras cepas resistentes de Streptococcus pneumoniae. Sin embargo, el problema no se tornó significativo hasta 1980 en Europa y 1990 en Estados Unidos. En los últimos años se ha observado además que este germen puede ser resistente a otros antibióticos betaláctamicos, a macrólidos e incluso a quinolonas de última generación. La resistencia a la penicilina se ha dividido convencionalmente en: intermedia, cuando la MIC es $>0.125 \mathrm{y}<1.0 \mathrm{mg} / 1$, y alta cuando es $>2.0 \mathrm{mg} / 1$. La situación actual en algunos países ha sido materia de discusión. En España, durante 1993 la tasa global de resistencia del S.pneumoniae fue de 35\% para la penicilina, $9 \%$ al cefotaxime, $13 \%$ a macrólidos y $33 \%$ a tetraciclinas. En el mismo período la resistencia a la penicilina fue de $70 \%$ en Corea, $25 \%$ en EU, $12 \%$ en Canadá y 29\% en Grecia. ${ }^{5}$ En Colombia, según un estudio de seguimiento en niños menores de cinco años realizado por el Instituto Nacional de Salud, se ha reportado una elevación de la sensibilidad disminuida a la penicilina del 10\% en 1994 al 49.4\% en 1999, posiblemente por el uso indiscriminado de los antibióticos y por la circulación de clones resistentes. No se conocen reportes sobre estudios en adultos a nivel nacional. ${ }^{9,10}$

A nivel mundial, la tasa de mortalidad de pacientes ambulatorios permanece baja con un rango menor del $5 \%$, pero en los que requieren hospitalización alcanza un 12\%. 2 En Colombia, en 1995 hubo 84.020 casos de NAC de los cuales 5.811 en Bogotá, 10 debido a que no es una enfermedad de reporte obligatorio. La información acerca de su incidencia se basa en estimaciones crudas según lo reportado por el Ministerio de Salud-Instituto Nacional de Salud. ${ }^{9}$

En el Hospital de San José se encontró durante los dos últimos años un alto porcentaje de NAC $(37.5 \%)$ como causa de hospitalización en el servicio de medicina interna. ${ }^{11}$ Nuestro objetivo, fue determinar cuáles agentes etiológicos están implicados en la NAC y su sensibilidad a los diferentes antibióticos utilizados, a través de la medición de la frecuencia de los gérmenes mediante cultivos de esputo, sangre o liquido pleural, además de determinar la sensibilidad de los microorganismos a los antibióticos utilizados. 


\section{Materiales y métodos}

Se realizó un estudio inicial describiendo la casuística del servicio de medicina interna del Hospital de San José desde abril 1 de 2003 hasta noviembre 30 de 2005. Los pacientes mayores de 15 años que ingresaron al servicio de urgencias con clínica sugestiva de neumonía, dada por la presencia de síntomas inespecíficos como escalofríos, fiebre, tos, disnea, expectoración mucopurulenta y dolor torácico tipo pleurítico acompañados o no de signos de consolidación y estertores finos al examen físico, y cumplieran con los criterios de hospitalización definidos en la clasificación de la Sociedad Americana de Tórax (Tabla 1 y 2). Se excluyeron los pacientes VIHSIDA, enfermedades hemato-oncológicas y en los que no fue posible la toma de al menos una muestra para estudio microbiológico.

A los incluidos en el estudio se les realizaron hemocultivos, así como gram y cultivo del esputo y del líquido pleural en caso de presentarlo. Los primeros fueron tomados por la enfermera jefe previo lavado de manos y limpieza del área de punción, en tres accesos venosos distintos, con diferentes jeringas y botellas, obteniendo muestras de 5 a $10 \mathrm{ml}$ de sangre, para ser llevados al laboratorio. Las botellas se colocaron en un equipo Bactec y cada quince minutos el equipo realizó lecturas. En caso de ser positivas se realizaron láminas para gram y subcultivos en agarsangre de cordero y agar-chocolate suplementado. Si el hemocultivo no era detectado como positivo en cinco días, se consideraba negativo. Para la rea- lización del gram y cultivo de esputo se tomó una muestra de la secreción expectorada por el paciente, ya fuera en forma espontánea o con la ayuda de terapia respiratoria, y se depositaron en un frasco estéril. A los pacientes que presentaron al ingreso signos radiológicos de derrame pleural se les realizó toracentesis para obtener una muestra del líquido.

El instrumento contaba con todos los datos de cada paciente: nombre, edad, género, comorbilidades, procedencia, tipo de NAC, antibiótico utilizado, complicaciones, gérmen(es) aislado(s), sensibilidad y estado de egreso del paciente. La información se recolectó al finalizar cada mes tomada de las historias clínicas del servicio de medicina interna. Para el análisis se realizaron tablas de frecuencia simples cruzando las variables disponibles con el programa SPSS e interpretando los resultados obtenidos.

\section{Resultados}

Desde abril 1 de 2003 a noviembre 30 de 2005 ingresaron al estudio un total de 159 pacientes, 63 hombres $(49.62 \%)$ y 96 mujeres $(60.37 \%)$ con un promedio de edad de 56 años y una desviación estándar de +/- 21 años. Al ingreso fueron clasificados como NAC IIIA 86 pacientes (54.1\%), NAC IIIB $66(41.5 \%), 6$ como NAC IVA (3.8\%) y uno como NAC IVB $(0.6 \%)$.

Dentro de las características demográficas se encontró que la ocupación más frecuente fue la de ama de

\section{Tabla I. Criterios de hospitalización - Sociedad Americana de Tórax*}

Historia clínica

Examen físico

Laboratorios
Edad >65 años, EPOC, DM IRC, (ICC), cirrosis, hospitalización previa <I año, sospecha de broncoaspiración, alteración estado mental, postesplenectomía, alcoholismo o desnutrición.

Frecuencia respiratoria $>30 / \mathrm{min}$, presión diastólica $60 \mathrm{~mm} \mathrm{Hg}$, sistólica $90 \mathrm{~mm} \mathrm{Hg}$, temperatura $>38.3^{\circ} \mathrm{C}\left(101^{\circ} \mathrm{F}\right)$, confusión, deterioro del estado de conciencia.

Leucocitos $<4 \times 10 / 3->10 \times 10 / 3, \mathrm{PaO} 2<60 \mathrm{~mm} \mathrm{Hg}$ o PaCO2 $>50$ mm Hg a FiO2 21\%, necesidad de ventilación mecánica, creatinina $>1.2 \mathrm{mg} / \mathrm{dl}$ o BUN $>20 \mathrm{mg} / \mathrm{dl}$ (>7 mmol/L), compromiso de más de un lóbulo, cavitaciones, presencia de derrame pleural, hematócrito $<30 \%$, hemoglobina $<9 \mathrm{~g} / \mathrm{dl}$, evidencia de sepsis o disfunción orgánica (acidosis metabólica, prolongación de tiempo de protrombina o tromboplastina, trombocitopenia, o aumento de productos de degradación de fibrinógeno $>$ I:40). 


\begin{tabular}{l|l}
\hline CLASIFICACIÓN & Tabla 2. Clasificación de NAC - Sociedad Americana de Tórax \\
\hline NAC I & $\begin{array}{l}\text { PaciNICIÓN } \\
\text { enfermedad. }\end{array}$ \\
\hline NAC II & $\begin{array}{l}\text { Pacientes ambulatorios con enfermedad cardiopulmonar y/o factores modificantes de la } \\
\text { enfermedad. }\end{array}$ \\
\hline NAC III A & $\begin{array}{l}\text { Pacientes hospitalizados que no requieren } \mathrm{UCl} \text { con enfermedad cardiopulmonar y/o factores } \\
\text { modificantes de la enfermedad. }\end{array}$ \\
\hline NAC III B & $\begin{array}{l}\text { Pacientes hospitalizados que no requieren } \mathrm{UCl} \text {, sin enfermedad cardiopulmonar y/o factores } \\
\text { modificantes de la enfermedad. }\end{array}$ \\
\hline NAC IVA & Pacientes hospitalizados que requieren $\mathrm{UCl}$ sin riesgo para Pseudomona aeruginosa. \\
\hline NAC IV B & Pacientes hospitalizados que requieren $\mathrm{UCl}$ con riesgo para Pseudomona aeruginosa. \\
\hline
\end{tabular}

casa en 68 (43\%), comerciantes 17 (10.8\%), seguido de otras actividades tales como vigilantes, bomberos, estudiantes, pensionados y profesionales. Nuestros pacientes provienen en su gran mayoría (139) de zonas urbanas $(87.4 \%)$ y $20(12.6 \%)$ rurales.

Las comorbilidades más frecuentes fueron la enfermedad pulmonar obstructiva crónica 43 pacientes (27.04\%), hipertensión arterial 15 (9.43\%), diabetes mellitus $10(6.28 \%$ ) y $42.76 \%$ no presentaron comorbilidad asociada (Tabla 3). A 102 se les realizaron hemocultivos $(64.2 \%)$, gram y cultivo de esputo a $122(76.7 \%)$ y cultivo de líquido pleural a solo cuatro pacientes $(2.5 \%)$.

\begin{tabular}{l|c|c}
\multicolumn{3}{c}{ Tabla 3. Frecuencia de comorbilidades } \\
\hline Ninguna & pacientes & $\%$ \\
\hline EPOC & 70 & 44,0 \\
\hline HTA & $4 I$ & 25,8 \\
\hline Diabetes mellitus & 15 & 9,4 \\
\hline Otros & 10 & 6,3 \\
\hline ICC & 10 & 6,3 \\
\hline Lupus eritematoso sistémico & 4 & 2,5 \\
\hline Enfermedad coronaria & 2 & 2,5 \\
\hline IRC & 2 & 1,3 \\
\hline Asma & 1 & 1,3 \\
\hline Total & 159 & 100,0 \\
\hline
\end{tabular}

En esputo solo se aislaron gérmenes en el 11.9\% de las muestras. El más frecuente fue candida albicans (5.0\%), seguido por Streptococcus pneumoniae (2.5\%), Staphylococcus aureus (1.9\%) y Streptococcus viridans y Harmophilus influenzae (1.3\%) (Tabla 4).

En el líquido pleural solo se cultivaron microorganismos en el $0.6 \%$ de las muestras y siempre fue Klebsiella pneumoniae.

Los hemocultivos fueron positivos en el 8.8\% de las muestras tomadas, siendo el más frecuente K.pneumoniae (3.1\%), seguido en menor porcentaje

\begin{tabular}{l|c|c|}
\multicolumn{3}{c}{ Tabla 4. Gérmenes aislados en esputo } \\
\hline Tipo de gérmen & Pacientes & $\%$ \\
\hline Candida albicans & 8 & 5,0 \\
\hline Streptococcus pneumoniae & 4 & 2,5 \\
\hline Staphylococcus aureus & 3 & 1,9 \\
\hline Streptococcus viridans & 2 & 1,3 \\
\hline Haemophilus influenzae & 2 & 1,3 \\
\hline Ninguno & 140 & 88,1 \\
\hline Total & 159 & 100,0 \\
\hline
\end{tabular}




\begin{tabular}{|c|c|c|}
\hline \multicolumn{2}{|c|}{ Tabla 5. Gérmenes aislados en hemocultivos } & $\%$ \\
\hline Klebsiella pneumoniae & Pacientes & 3,1 \\
\hline Streptococcus pneumoniae & 5 & 2,5 \\
\hline Staphylococcus aureus & 4 & 1,3 \\
\hline Staphylococcus hominis & 2 & 1,3 \\
\hline Pseudomona aeruginosa & 2 & 0,6 \\
\hline Ninguno & 1 & 91,2 \\
\hline Total & 145 & 100,0 \\
\hline
\end{tabular}

\begin{tabular}{|c|c|c|c|c|c|c|c|c|c|c|}
\hline \multirow{3}{*}{ Complicación } & \multicolumn{8}{|c|}{ Tipo de NAC } & \multirow{2}{*}{\multicolumn{2}{|c|}{ Total }} \\
\hline & \multicolumn{2}{|c|}{ NAC IIIA } & \multicolumn{2}{|c|}{ NAC IIIB } & \multicolumn{2}{|c|}{ NAC IVA } & \multicolumn{2}{|c|}{ NAC IVB } & & \\
\hline & Recuento & $\%$ & Recuento & $\%$ & Recuento & $\%$ & Recuento & $\%$ & Recuento & $\%$ \\
\hline Absceso pulmonar & I & 0,6 & & & & & & & 1 & 0,6 \\
\hline Empiema & 1 & 0,6 & I & 0,6 & & & & & 2 & 1,3 \\
\hline Sepsis & 1 & 0,6 & 5 & 3,1 & 2 & 1,3 & 1 & 0,6 & 9 & 5,7 \\
\hline Sin respuesta & & & & 2 & 1,3 & & & & 2 & 1,3 \\
\hline Muerte & 2 & $\mathrm{I}, 3$ & & & 1 & 0.6 & & & 3 & 1.9 \\
\hline Ninguna & 79 & 49,7 & 57 & 35,8 & 3 & 1,9 & & & 139 & 87,4 \\
\hline Otros & 2 & $\mathrm{I}, 3$ & $\mathrm{I}$ & 0,6 & & & & & 3 & 1,9 \\
\hline Total & 86 & 54,1 & 66 & $4 I, 5$ & 6 & 3,8 & 1 & 0,6 & 159 & 100,0 \\
\hline
\end{tabular}

por S. pneumoniae, Staphylococcus aureus, Stafilococcus hominis y Pseudomona aeruginosa (Tabla 5). En nuestros pacientes, la complicación más común fue sepsis en nueve pacientes (5.7\%), seguida de muerte en tres (1.9\%). Se encontró que el 87.4\% no presentó ninguna complicación, el $98.1 \%$ egresó vivo de la institución con resolución de su cuadro infeccioso (Tabla 6).

\section{Discusión}

En este estudio descriptivo se tuvo como principal limitación el inadecuado diseño del instrumento que no permitió recolectar en forma adecuada los datos de sensibilidad y resistencia antimicrobiana, por lo cual no pudieron ser analizados.
Entre los hallazgos es importante resaltar que los gérmenes en la población estudiada coinciden con los descritos en la literatura como Streptococcus pneumoniae y Staphylococcus aureus, sin ser estos en nuestro estudio los de mayor porcentaje, ya que en los hemocultivos fue la Klebsiella pneumoniae la que se encontró con mayor frecuencia (3.1\%) y en esputo la Candida albicans (5.0\%), lo que no era esperado de acuerdo con la literatura. ${ }^{12}$ Entre las comorbilidades asociadas a NAC es de gran importancia resaltar que la EPOC se encontró en un $27 \%$ de los pacientes, sugiriéndola como un importante factor de riesgo, ${ }^{13}$ seguida de DM en un $6.3 \%$.

Aparentemente por la respuesta clínica obtenida, las pocas complicaciones, la baja mortalidad y teniendo en cuenta el inicio empírico de los antibióticos, se podría plantear la baja resistencia antimicrobiana de 
estos agentes etiológicos, pero se requieren estudios adicionales para confirmar nuestra hipótesis, dadas las limitaciones y así poder considerar como primera línea de elección el uso de ampicilina sulbactam y la combinación Ceftriaxona más claritromicina.

\section{Referencias}

1. Pierre Y. Community-acquired pneumonia. Clin Infect Dis. 2001;80:75-87.

2. American Thoracic Society. Guidelines for the management of Adults with Community-acquired Pneumonia. Crit Care Med. 2001;163:1730-1754.

3. Levinson ME. Neumonía, comprendidas las infecciones pulmonares necrotizantes. En: Braunwald E, Fauci AS, Kasper D, Hauser S, Longo D, Jameson J., editores. Harrison Principios de Medicina Interna. 15 ed. 2002:1729-1740.

4. Chaparro C. Manejo de las nemonías de los adultos. En: Camacho F, Awad, C, editores. Enfermedades del Tórax. [Bogotá]: Hospital Santa Clara; [1992] p. 226-243.

5. Caballero A. Infecciones del aparato respiratorio. En: Chalem F, Escandón J, Campos J, Esguerra R. Medicina interna. $3^{\text {a }}$. ed. 1998. p. 1457-1462.

6. Barlett. J. Practice Guidelines for the management of Community-Acquired Pneumonia in adults. Clin Infect Dis. 2000 Sep; 31: 347-82.
7. Niederman MS. Infecciones respiratorias. Clínicas Médicas de Norteamérica. 2001;6:1283-1549.

8. Mandell G, Benneth J, Dolin R. Principles and practice of infectious diseases, 6th. ed. [Philadelphia] : Churchill Livingstone; 2002.

9. Ministerio de salud, Instituto Nacional de Salud. Vigilancia por el laboratorio de Streptococcus pneumoniae aislado de procesos invasores en niños menores de 5 años: actualización de datos 1994-1999. Inf Epidemiol Nac. 2000 May; 5 (9): 131-135.

10. Mandell LA, Marrie TJ, Grossman RF, Chow AW, Hyland $\mathrm{RH}$, Canadian guidelines for the initial management of community-acquired pneumonia: an evidence-based update by the Canadian Infectious Disease Society and the Canadian Thoracic Society. Can Respir J. 2000 SepOct;7(5):371-82.

11. Martínez C, Jaimes F, Montúfar F, et al. Proceso de atención y prescripción en neumonía adquirida en la comunidad en hospitales universitarios en Colombia [artículo en Internet] [citado 2006 Jun 15] Disponible en: http://www.encolombia.com/medicina/neumologia/neumologia15403-proceso. htm.

12. Etiology of Community-Acquired Pneumonia. Clin Chest Med. 2005 Mar;26(1):47-55.

13. Hospital de San José. Reporte anual hospitalización Departamento de Medicina Interna. Bogotá: Hospital de San José; 2001 -2002.

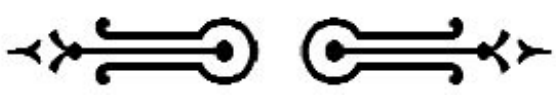

